\title{
Direct Evidence of Self-catalysis effect in ZnO Nanobelt Growth
}

\author{
Yong Ding, Yaguang Wei, Cheng Li, Zhong Lin Wang
}

School of Materials Science and Engineering, Georgia Institute of Technology, Atlanta, GA 30332-0245

Uniformly aligned $\mathrm{ZnO}$ nanobelt arrays have been grown on a (0001) GaN substrate without foreign catalyst such as $\mathrm{Au}$. The nanobelts mainly grew along [01 $\overline{1} 3$ ] at beginning when the temperature was relatively low, then switched to $[01 \overline{1} 0]$ at high temperature, and finally switched to [01 13 3] when the temperature was lowed. The alignment of the nanobelts was due to not only the epitaxial orientation relationship with the substrate but more importantly the presence of metallic $\mathrm{Zn}$ nanoparticles at the tip of the $\mathrm{ZnO}$ nanobelts, which were produced by reduction of $\mathrm{ZnO}$ source material. The $\mathrm{Zn}$ nanoparticle has a fixed orientation relationship with the $\mathrm{ZnO}$ nanobelt, indicating that the growth follows the self-catalyzed vapor-liquid-solid process.

Each belt we examined by TEM is only part of the whole nanostructure grown from the substrate. Different sections of nanobelts can be identified using their morphologies. During vapor deposition process, a seed layer was first epitaxially grown on the substrate, and then $\mathrm{ZnO}$ nanobelts grew up form such a thin film layer. Therefore, the nanobelt in Figure 1a, which looks like peeled off from a thin film layer, can be considered as the root of nanobelt binding with the $\mathrm{GaN}$ substrate. Figure $2 \mathrm{~b}$ is a HRTEM image of the rectangle area in Figure 1a. The growth direction can be uniquely identified as [01 $\overline{1} 3$ ], which corresponds to the starting growth direction. In figure 1c, an arrow points to a particle at the growth front of the nanobelt. The uniform contrast of the nanobelt and the inserted SAED pattern indicate that it is a single crystal with two growth directions along $[01 \overline{1} 0]$ and [01 $\overline{1} 3]$, respectively. The turning points of growth directions between [01 $\overline{1} 0]$ and [01 $\overline{1} 3]$ are circled in Figure $3 \mathrm{c}$.

Figure 2a confirms that the $\mathrm{ZnO}$ nanobelts grew along [01 13 3] at the tip. From the analysis above, we can see that the NBs initially grew along [01 $\overline{1} 3]$, then switched to [01 $\overline{1} 0]$ during growth, and then the final part of the $\mathrm{ZnO} N B s$ took the [01 $\overline{1} 3$ ] as its growth direction. Also in Fig. 2, it shows a single crystalline $\mathrm{Zn}$ particle that is covered by a thin $\mathrm{ZnO}$ layer, which was formed due to the oxidation of Zn when exposed to air. The particle's SAED pattern is displayed in Figure 4b, which is composed of two patterns; one is $\mathrm{ZnO}$ [2 $\overline{1} \overline{1} 0]$, and another one is the $\mathrm{Zn}$ [0001] pattern as indicated by white arrowheads. The lattice match configuration can be described as $(2 \overline{1} \overline{1} 0)_{\mathrm{ZnO}}\left|(0001)_{\mathrm{Zn}},[0001]_{\mathrm{ZnO}}\right|[01 \overline{1} 0]_{\mathrm{Zn}}$. The observation of $\mathrm{Zn}$ particle and its lattice match to $\mathrm{ZnO}$ NB indicates the existence of a self-catalyzed growth process. In our experiment the only source material was pure $\mathrm{ZnO}$ powder and there was no foreign catalyst deposited on the substrate. The $\mathrm{Zn}$ self-catalyzed growth of $\mathrm{ZnO}$ nanowires/nanobelts has been proposed previously.[2] Our experiment gives the direct evidence of such self-catalyzed growth using $\mathrm{ZnO}$ decomposed $\mathrm{Zn}$ particle.

Reference:

[1] Y. G. Wei, Y. Ding, C. Li, S. Xu, Jae-Hyun Ryo, R. Dupuis, A. K. Sood, D. L. Polla, and Z. L. Wang, J. Phys. Chem., 112 , 18935 (2008) .

[2] Z. L Wang, X. Y. Kong, J. M. Zuo, Phys. Rev. Lett., 91, 185502 (2003). 


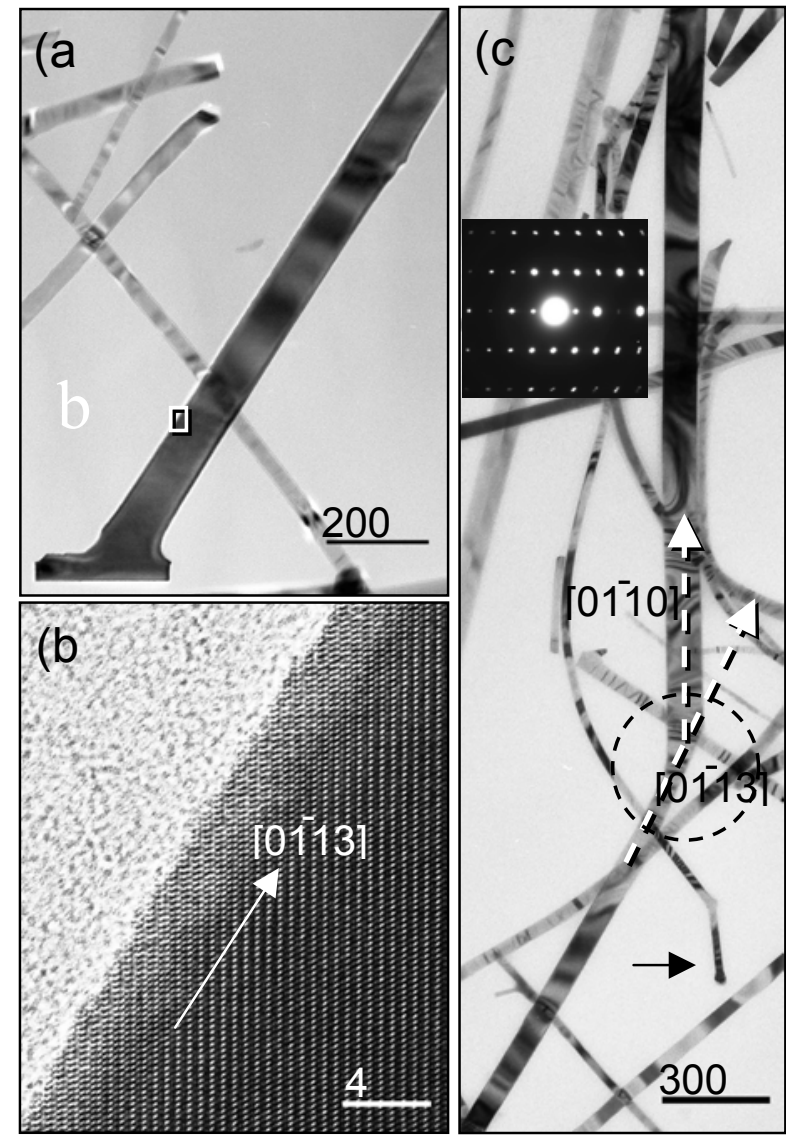

Fig. 1. (a) Low-magnification TEM image of the root of a $\mathrm{ZnO}$ nanobelt in contact with the GaN substrate. (b) High-resolution TEM image from the rectangular area in (a). The root of the nanobelt grows along [01 13 3] direction. (c) TEM image shows the turning point (circled area) of the growth direction from [01 $\overline{1} 3]$ to [01 $\overline{1} 0]$. Inset: the SAED pattern of the largest nanobelt shown in (c).

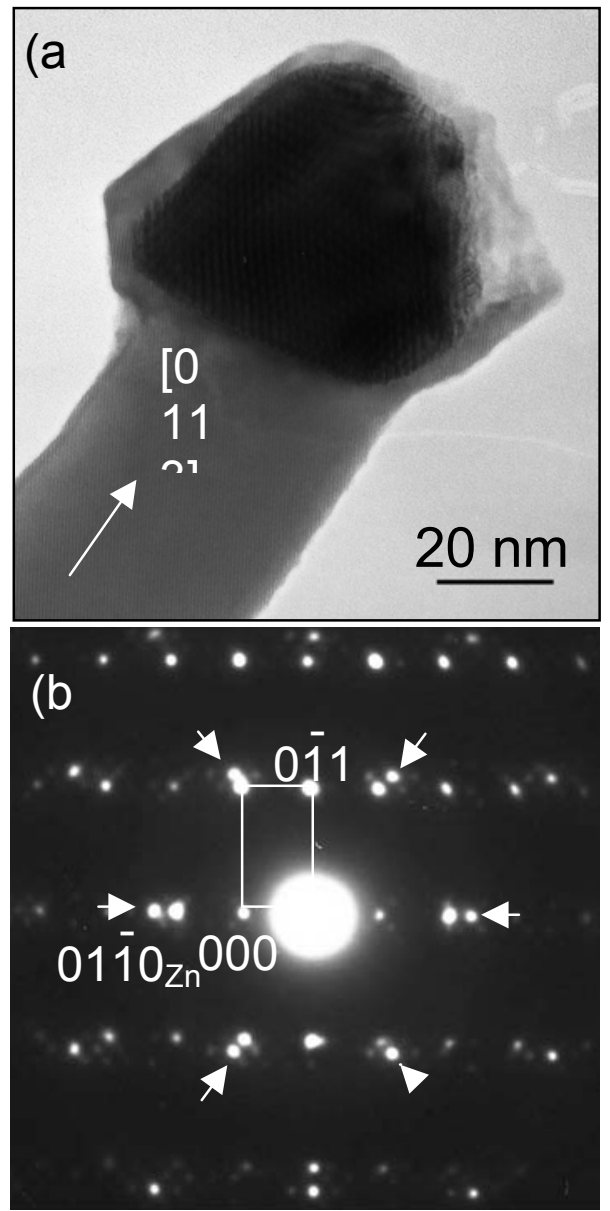

Fig. 2. High-resolution TEM image (a) and corresponding SAED pattern (b) of a $\mathrm{ZnO}$ nanobelt with a $\mathrm{Zn}$ particle located at its tip. Note the relative rotation of the diffraction patterns in reference to the image was not adjusted for nice display purpose. 